\title{
Non-perturbative renormalisation for overlap fermions
}

Martin Gürtler $^{a}$, Roger Horsley ${ }^{b}$, Paul E. L. Rakow ${ }^{c}$, Charles J. Roberts ${ }^{* c}$, Gerrit Schierholz ${ }^{a d}$ and Thomas Streuer ${ }^{a e}$

a John von Neumann-Institut für Computing NIC, Deutsches Elektronen-Synchrotron DESY, 15738

Zeuthen, Germany

${ }^{b}$ School of Physics, University of Edinburgh, Edinburgh EH9 3JZ, UK

${ }^{c}$ Theoretical Physics Division, Department of Mathematical Sciences, University of Liverpool, Liverpool L69 3BX, UK

${ }^{d}$ Deutsches Elektronen-Synchrotron DESY, 22603 Hamburg, Germany

e Institut für Theoretische Physik, Freie Universität Berlin, 14196 Berlin, Germany

${ }^{*}$ E-mail: cjr@liv.ac.uk

\section{QCDSF Collaboration}

Using non-perturbative techniques we have found the renormalisation factor, $Z$, in the RI-MOM scheme for quark bilinear operators in quenched QCD. We worked with overlap fermions using the Lüscher-Weisz gauge action. Our calculation was performed at $\beta=8.45$ at a lattice spacing of $1 / a=2.1 \mathrm{GeV}$ using a value of $\rho=1.4$. Our results show good agreement between the vector and the axial vector in the zero mass limit. This shows that overlap fermions have good chiral properties. To attempt to improve the discretisation errors in our results we subtracted the $\mathscr{O}\left(a^{2}\right)$ terms in one-loop lattice perturbation theory from the Monte Carlo Green functions. In particular we paid attention to the operators for the observable $\langle x\rangle$. We found a value for the renormalisation constants $Z_{v_{2 b}}^{\overline{\mathrm{MS}}}$ and $Z_{v_{2 a}}^{\overline{\mathrm{MS}}}$ just less than 1.9 at $\mu=1 / a=2.1 \mathrm{GeV}$.

XXIIIrd International Symposium on Lattice Field Theory

25-30 July 2005

Trinity College, Dublin, Ireland

${ }^{*}$ Speaker. 


\section{Introduction}

In a renormalisation scheme $\mathscr{S}$ a general renormalised operator is given by

$$
\mathscr{O}^{\mathscr{S}}(M)=Z_{\mathscr{O}}^{\mathscr{S}}(M) \mathscr{O}^{\text {bare }},
$$

where $M$ is the scale. We have followed the Rome-Southampton method [1] for determining the renormalisation constants of various lattice operators non-perturbatively in the regularisation invariant (RI-MOM) scheme. In particular we have paid attention to the operators for the observable $\langle x\rangle$.

We have calculated the amputated Green functions, $\Lambda_{\mathscr{O}}$, for such operators with overlap fermions using the tadpole improved Lüscher-Weisz gauge action in the quenched approximation. Our calculation was performed at $\beta=8.45$ at a lattice spacing of $1 / a=2.1 \mathrm{GeV}$ using a value of $\rho=1.4$. These are the same parameters that we used in [2]. The gauge was fixed to the Landau gauge. We used a momentum source which results in smaller errors [3].

In both the RI-MOM and RI'-MOM schemes we require

$$
\left.\frac{Z_{\mathscr{O}}^{\mathscr{S}}}{Z_{\psi}^{\mathscr{S}}} \Lambda_{\mathscr{O}}^{\text {bare }}\right|_{p^{2}=\mu^{2}}=\Lambda_{\mathscr{O}}^{\text {tree }}+\text { other Dirac structures }
$$

where $Z_{\psi}^{\mathscr{S}}$ is the field renormalisation constant. So by measuring $\Lambda_{\mathscr{O}}^{\text {bare }}$ on the lattice we can find $Z_{\mathscr{O}}^{\mathscr{S}} / Z_{\psi}^{\mathscr{S}}$, but we need to eliminate $Z_{\psi}^{\mathscr{S}}$.

The two schemes differ in their definition of $Z_{\psi}^{\mathscr{S}}$. In the $\mathrm{RI}^{\prime}$-MOM scheme

$$
\left.Z_{\psi}^{\mathrm{RI}} S\right|_{p^{2}=\mu^{2}}=S^{\text {tree }},
$$

where $S$ is the Landau gauge propagator. In the RI-MOM scheme

$$
\left.\frac{1}{Z_{\psi}^{\mathrm{RI}}} \frac{1}{16 N_{C}} \sum_{v} \operatorname{Tr}\left[\gamma_{v} \Lambda_{V_{v}^{C}}^{\text {bare }}\right]\right|_{p^{2}=\mu^{2}}=1,
$$

where $V^{C}$ is the conserved vector current. Both present difficulties for overlap fermions. The propagator has large $\mathscr{O}\left(a^{2}\right)$ artefacts, which cannot be easily eliminate, and $V^{C}$ is hard to define or measure for overlap fermions.

Fortunately, we know that the conserved vector current, $V^{C}$, is proportional to the local vector current, $V$, so we can find $Z_{\psi}^{\mathrm{RI}}$ from $\Lambda_{V}^{\text {bare }}$. Because it is conserved, $Z_{V^{C}}=1$. So

$$
\frac{1}{Z_{\psi}^{\mathrm{RI}}} \Lambda_{V^{C}}^{\text {bare }}=\frac{Z_{V}}{Z_{\psi}^{\mathrm{RI}}} \Lambda_{V}^{\text {bare }} \quad \Leftrightarrow \quad \Lambda_{V^{C}}^{\text {bare }}=Z_{V} \Lambda_{V}^{\text {bare }} .
$$

This allows us to measure $Z_{V}$ non-perturbatively by looking at the proton's charge and baryon number [2]. At $\beta=8.45, Z_{V}=1.416$.

So, if $Z_{V}$ is known, we can combine these relations to get

$$
Z_{\psi}^{\mathrm{RI}}=\left.Z_{V} \frac{1}{16 N_{C}} \sum_{v} \operatorname{Tr}\left[\gamma_{v} \Lambda_{V_{V}^{C}}^{\text {bare }}\right]\right|_{p^{2}=\mu^{2}},
$$


which allows us to find $Z_{\mathscr{O}}^{\mathrm{RI}}$ alone.

The $v_{2 b}$ and $v_{2 a}$ operators are defined as $\bar{\psi}\left(\gamma_{\mu} T_{\mu \nu} D_{v}\right) \psi$, where $T_{\mu \nu}$ is a symmetric, traceless tensor. For the $v_{2 b}$ operators $T_{\mu \nu}$ is diagonal. For the $v_{2 a}$ operators it is off-diagonal. These operators are used to find $\langle x\rangle$ in a hadron. This observable is important in deep inelastic scattering.

The running of the renormalised operator with the scale $M$ is controlled by the $\beta$ and $\gamma$ functions in the renormalisation group equation. These are defined as the scale derivatives of the renormalised coupling and the renormalisation constant:

$$
\begin{aligned}
& \left.\beta^{\mathscr{S}}\left(g^{\mathscr{S}}(M)\right) \equiv \frac{\partial g^{\mathscr{S}}(M)}{\partial \log M}\right|_{\text {bare }}, \\
& \left.\gamma_{\mathscr{O}}^{\mathscr{S}}\left(g^{\mathscr{S}}(M)\right) \equiv \frac{\partial \log Z_{\mathscr{O}}^{\mathscr{S}}(M)}{\partial \log M}\right|_{\text {bare }},
\end{aligned}
$$

where the bare parameters are held constant. These functions are given perturbatively as power series expansions in the coupling constant. Thus

$$
\begin{aligned}
& \beta^{\mathscr{S}}(g)=-b_{0} g^{3}-b_{1} g^{5}-b_{2}^{\mathscr{S}} g^{7}-b_{3}^{\mathscr{S}} g^{9}-\cdots, \\
& \gamma_{\mathscr{O}}^{\mathscr{S}}(g)=d_{\mathscr{O} 0} g^{2}+d_{\mathscr{O} 1}^{\mathscr{S}} g^{4}+d_{\mathscr{O} 2}^{\mathscr{S}} g^{6}+d_{\mathscr{O} 3}^{\mathscr{S}} g^{8}+\cdots .
\end{aligned}
$$

The $\beta$ function is now known to four loops in the $\overline{\mathrm{MS}}$ scheme [4]. For the scalar and pseudoscalar operators, the $\gamma$ function is known to four loops in the $\overline{\mathrm{MS}}$ and RI-MOM schemes [4]. For the tensor, pseudotensor, $v_{2 b}$ and $v_{2 a}$ operators it is known to three loops $[5,6]$ in these schemes.

We found the renormalisation group invariant (RGI) renormalisation constant using the relation

$$
\mathscr{O}^{R G I} \equiv \Delta Z_{\mathscr{O}}^{\mathscr{S}}(M) O(M)=\Delta Z_{\mathscr{O}}^{\mathscr{S}}(M) Z_{\mathscr{O}}^{\mathscr{S}}(M) \mathscr{O}^{\text {bare }},
$$

where

$$
\left[\Delta Z_{\mathscr{O}}^{\mathscr{S}}(M)\right]^{-1}=\left[2 b_{0} g^{\overline{\mathrm{MS}}}(M)^{2}\right]^{-d_{\mathscr{O}} / 2 b_{0}} \exp \left\{\int_{0}^{g^{\overline{\mathrm{MS}}}(M)} d \xi\left[\frac{\gamma_{\mathscr{O}}^{\mathscr{S}}(\xi)}{\beta^{\overline{\mathrm{MS}}}(\xi)}+\frac{d_{\mathscr{O} 0}}{b_{0} \xi}\right]\right\} .
$$

We used anomalous dimensions in the RI-MOM scheme and a scale of $a^{2} p^{2}$. We then converted the RGI renormalisation constant to the $\overline{\mathrm{MS}}$ scheme at the scale $a \mu=1$ using a similar relation involving the $\overline{\mathrm{MS}}$ anomalous dimensions.

Even after this our data still contained lattice artefacts, especially at large $p^{2}$. To reduce these we calculated the same Green functions in lattice perturbation theory to one loop. We subtracted off the terms of leading order in the lattice spacing and regarded the remaining quantity of $\mathscr{O}\left(a^{2}\right)$ as a lattice artefact. This lattice artefact we subtracted from the Monte Carlo Green function and we compared the results before and after this subtraction. When finding the lattice perturbation theory result we used a coupling $g^{\overline{\mathrm{MS}}}(k p)$ and experimented with different values of $k$.

\section{Results}

Firstly we tested to see whether the Green functions found using overlap fermions had better chiral properties than those found using conventional Wilson and clover fermions. Figure 1 shows 


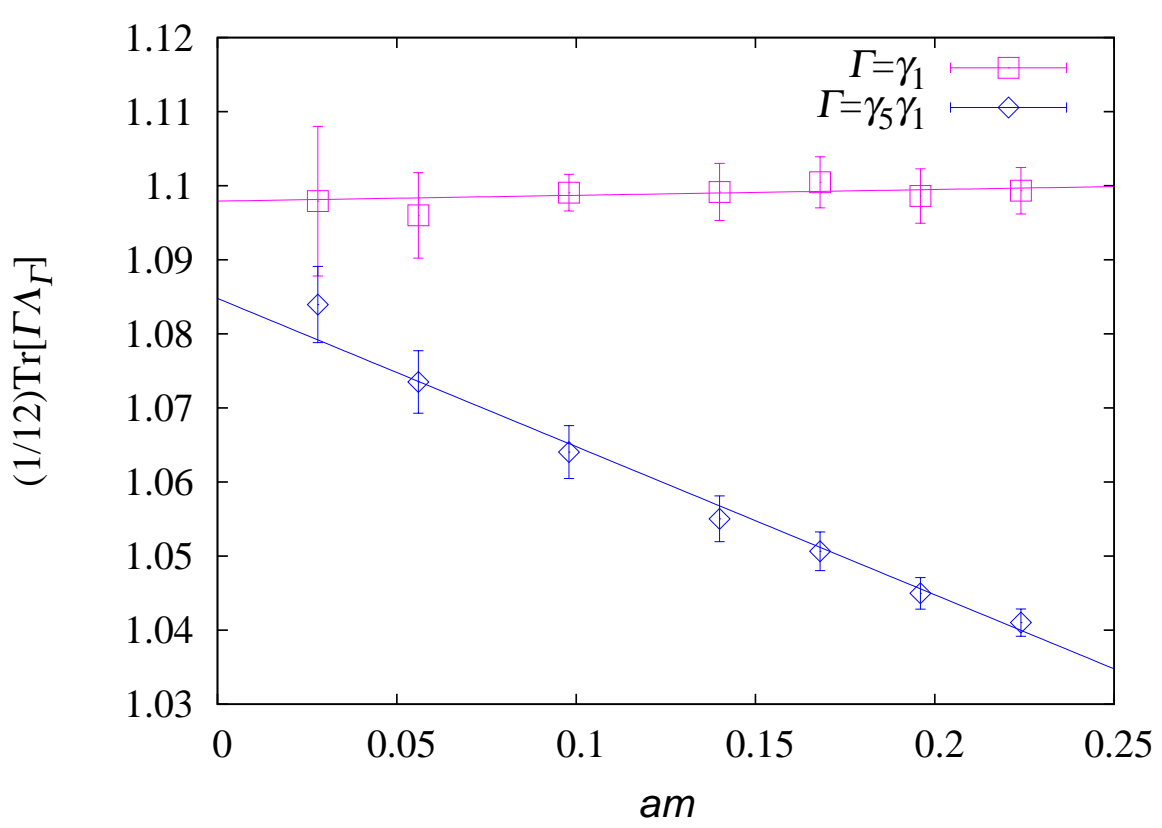

Figure 1: Here am is the bare mass in lattice units. Our extrapolation to zero mass for a vector and an axial vector shows that there is good agreement between the two at zero mass for $a^{2} p^{2}=0.529$. At higher $p^{2}$ the agreement improves.
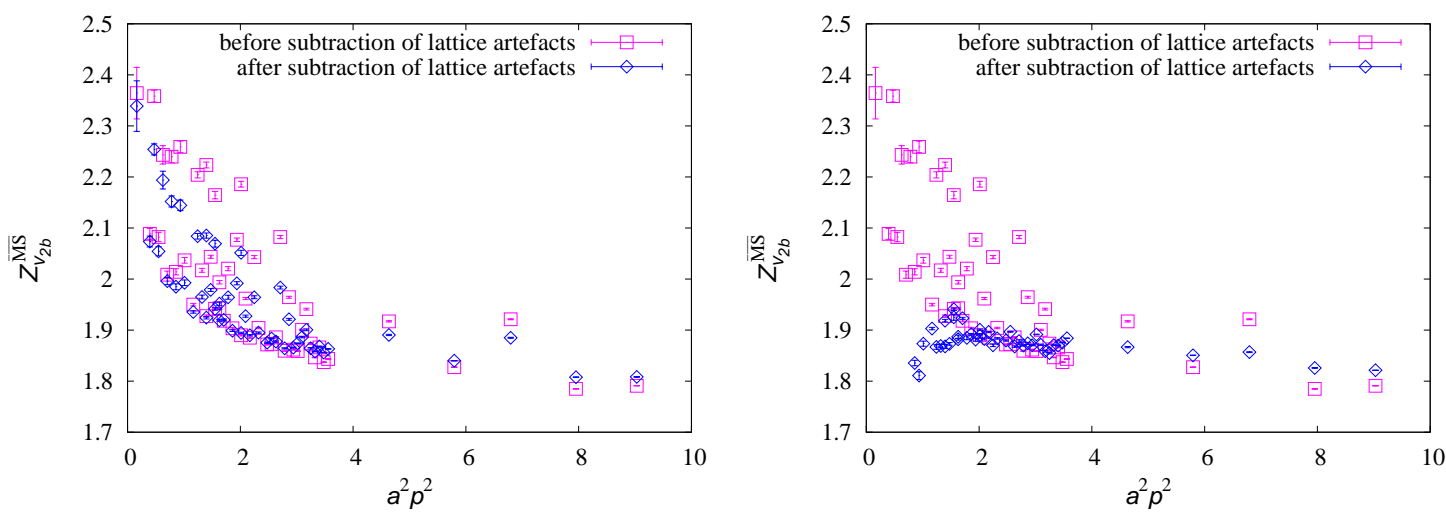

Figure 2: The operator shown here is $\bar{\psi}\left[\gamma_{4} D_{4}-1 / 3\left(\gamma_{1} D_{1}+\gamma_{2} D_{2}+\gamma_{3} D_{3}\right)\right] \psi$, where 4 is the time direction. We obtained a value of the renormalisation constant $Z_{v_{2 b}}^{\overline{\mathrm{MS}}}$ just less than 1.9 at $\mu=1 / a=2.1 \mathrm{GeV}$. The coupling we used to calculate the artefacts on the left was $g^{\overline{\mathrm{MS}}}(p)$. The coupling we used to calculate the artefacts on the right was $g^{\overline{\mathrm{MS}}}(k p)$, where $k=0.22$. There are less points after subtraction of lattice artefacts since this coupling is not defined for the lower momenta. 


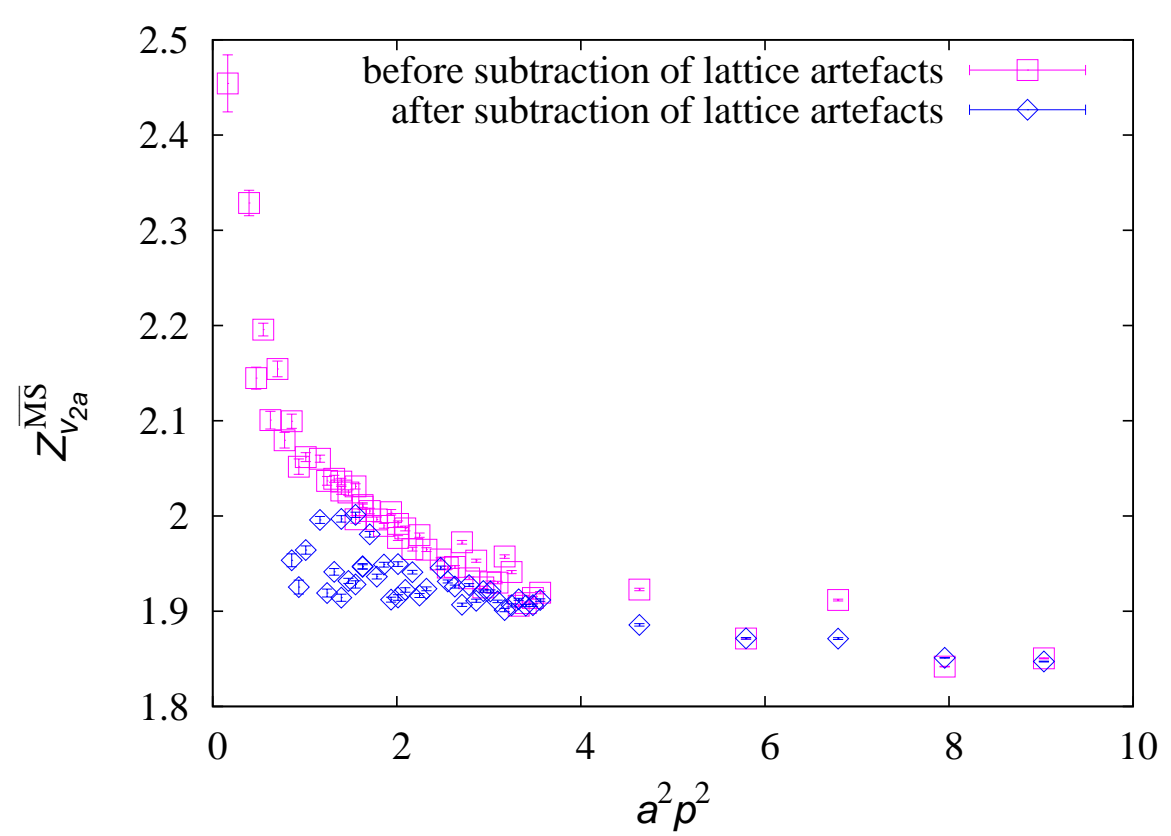

Figure 3: The operator shown here is $\bar{\psi}\left[1 / \sqrt{2}\left(\gamma_{1} D_{4}+\gamma_{4} D_{1}\right)\right] \psi$, where 4 is the time direction. We obtained a value of the renormalisation constant $Z_{v_{2 a}}^{\overline{\mathrm{MS}}}$ just less than 1.9 at $\mu=1 / a=2.1 \mathrm{GeV}$. The coupling we used to calculate the artefacts was $g^{\overline{\mathrm{MS}}}(k p)$, where $k=0.22$. There are less points after subtraction of lattice artefacts since this coupling is not defined for the lower momenta.

our extrapolation to zero mass for a vector and an axial vector at $a^{2} p^{2}=0.529$. At this momentum the agreement between the two results is quite good at zero mass. At higher values of the momentum the agreement improves further. This is not the case with Wilson and clover fermions and shows that overlap fermions have a proper chiral symmetry.

Figure 2 shows a plot of $Z_{v_{2 b}}^{\overline{\mathrm{MS}}}$ against $a^{2} p^{2}$. There is a scale dependence because of the discretisation of the lattice. In particular, there is scatter, because the result depends on the directionality of the momentum as well as its magnitude. This is entirely an artefact of the lattice. We would expect this plot to be a perfect plateau because when we converted to the RGI $Z$ at a scale $a^{2} p^{2}$ this should have removed the scale dependence.

To attempt to improve our plateau we calculated the $\mathscr{O}\left(a^{2}\right)$ terms for the same Green functions in one loop lattice perturbation theory and subtracted them from the Monte Carlo Green functions. On the left in figure 2 we used a coupling $g^{\overline{\mathrm{MS}}}(p)$ for the lattice perturbation theory calculation. Our subtraction of the lattice artefacts gives some improvement in the right direction, but still not enough.

It is possible to rescale the coupling to $g^{\overline{\mathrm{MS}}}(k p)$ in one loop lattice perturbation theory. This is because the difference between $g^{\overline{\mathrm{MS}}}(p)$ and $g^{\overline{\mathrm{MS}}}(k p)$ is $\mathscr{O}\left(g^{4}\right)$. It is not possible to tell the scale from one loop lattice perturbation theory alone. Thus we tried experimenting with rescaled couplings using different values of $k$ to see if this would improve our results.

Plots for two of the operators we considered are shown in figure 2 on the right and figure 3. These use a coupling $g^{\overline{\mathrm{MS}}}(k p)$, where $k=0.22$, in calculating the lattice artefacts. It is necessary 
to use a value of $k$ as small as this to significantly improve the plateau. Although at first sight the factor $k$ might seem rather small, it is worth noting that the $\Lambda$ parameters which set the scale for different gauge actions can vary a great deal (see for example Table 6 of [7]). So perhaps such a large rescaling is not unreasonable.

Finally, from figure 2 and figure 3 , we obtained a value of the renormalisation constants $Z_{v_{2 b}}^{\overline{\mathrm{MS}}}$ and $Z_{v_{2 a}}^{\overline{\mathrm{MS}}}$ just less than 1.9, which is in agreement with the results of [8].

\section{Conclusion}

The good agreement between the results for the vector and the axial vector in the zero mass limit shows that overlap fermions have a proper chiral symmetry. There would not be such an agreement with Wilson or clover fermions.

We can improve our plateau in our results in figure 2 and figure 3 quite significantly by choosing an appropriate value of $k$. We intend to investigate this further by doing a fit for the best value of $k$ and also by experimenting with different values of $\beta$ and seeing if the same value of $k$ also works there.

\section{References}

[1] G. Martinelli, C. Pittori, C. T. Sachrajda, M. Testa and A. Vladikas, A general method for non-perturbative renormalization of lattice operators, Nucl. Phys. B445 (1995) 81-105 [hep-lat/9411010]

[2] D. Galletly, M. Gürtler, R. Horsley, B. Joó, A. D. Kennedy, H. Perlt, B. J. Pendleton, P. E. L. Rakow, G. Schierholz, A. Schiller and T. Streuer, Quark spectra and light hadron phenomenology from overlap fermions with improved gauge field action, Nucl. Phys. Proc. Suppl. 129 (2004) 456-467 [hep-lat/0310028]

[3] M. Göckeler, R. Horsley, H. Oelrich, H. Perlt, D. Petters, P. E. L. Rakow, A. Schäfer, G. Schierholz and A. Schiller, Non-perturbative renormalisation of composite operators in lattice QCD, Nucl. Phys. B544 (1999) 699-733 [hep-lat/9807044]

[4] K. G. Chetyrkin and A. Rétey, Renormalization and running of quark mass and field in the regularization invariant and $\overline{M S}$ schemes at three and four loops, Nucl. Phys. B583 (2000) 3-34 [hep-ph/9910332]

[5] J. A. Gracey, Three loop anomalous dimension of non-singlet quark currents in the RI' scheme, Nucl. Phys. B662 (2003) 247-278 [hep-ph/ 0304113$]$

[6] J. A. Gracey, Three loop anomalous dimension of the second moment of the transversity operator in the $\overline{M S}$ and RI' schemes, Nucl. Phys. B667 (2003) 242-260 [hep-ph/ 0306163 ]

[7] R. Horsley, H. Perlt, P. E. L. Rakow, G. Shierholz and A. Schiller, One-loop renormalisation of quark bilinears for overlap fermions with improved gauge actions, Nucl. Phys. B693 (2004) 3-35, Erratum ibid. B713 (2005) 601-606 [hep-lat/0404007]

[8] D. Galletly, M. Gürtler, R. Horsley, K. Koller, V. Linke, P. E. L. Rakow, G. Schierholz and T. Streuer, Nucleon structure from quenched overlap fermions, PoS (LAT2005) 363 